\title{
CALCULATION OF PULSED KICKER MAGNETIC FIELD ATTENUATION INSIDE BEAM CHAMBERS
}

\author{
S. H. Kim \\ January 8, 2001
}

\section{Introduction and Summary}

The ceramic beam chambers in the sections of the kicker magnets for the beam injection and extraction in the Advanced Photon Source (APS) are made of alumina. The inner surface of the ceramic chamber is coated with a conductive paste. The choice of coating thickness is intended to reduce the shielding of the pulsed kicker magnetic field while containing the electromagnetic fields due to the beam bunches inside the chamber, and minimize the Ohmic heating due to the fields on the chamber [1]. The thin coating generally does not give a uniform surface resistivity for typical dimensions of the ceramic chambers in use. The chamber cross section is a circular or an elliptic shape. The chamber or its wall thickness refers to the conductive coating in the following sections.

This note calculates the penetration of the kicker magnetic field inside the beam chamber. The kicker field is assumed to be a half-sine pulse and be spatially uniform over the chamber dimensions. The purpose of the calculation is to be able to deduce the average surface resistivity of a chamber by fitting the measured magnetic field data with the calculation inside the chamber. In the following section, assuming that the coating thickness $d$ is much smaller than the classical skin depth $\delta$, the penetrated field inside the chamber is calculated by subtracting the shielding field due to the eddy currents. In Section 3, for the kicker fields parallel and perpendicular to the axis of a circular beam chamber, the fields inside the chamber with an arbitrary wall thickness are calculated. For both directions of the kicker fields, the approximations made for $d<<\delta$ achieve the same results as given in Section 2. For elliptic chambers, the calculations for the vector potentials are not completed because of the tedious approximation procedure with Mathieu functions. Instead, the results in Section 2 and the time constants calculated for the elliptic geometries in Table 1 could be used for the purpose of this note.

\section{Simplified Solution}

The two-dimensional cross section of a circular cylinder is depicted in Fig. 1. The conducting-wall thickness, $d=b-a$, is greatly exaggerated compared to its inner and outer radii, $a$ and $b$, and the main body of the chamber (ceramic) is not shown. The kicker magnetic field $B_{y}(t)$ is applied perpendicular to the chamber axis. Neglecting the eddy current distributions near both ends of the chamber, the eddy currents in the chamber are assumed to be parallel to its axis. For a simple calculation of the eddy current shielding, we will follow the textbook by Smythe [2]. The eddy currents due to the inducing kicker field $B_{y}\left(t^{\prime}\right)$, at time $t^{\prime}$ before the present time $t$, reduce the field inside the chamber at time $t$. The eddy currents have been decaying out exponentially from the time $t^{\prime}$ with a time constant $\tau$. Then the field inside the chamber at time $t$, $B_{i}(t)$, may be calculated by subtracting the shielding field due to the eddy currents from the kicker field $B_{y}(t)$ : 


$$
\begin{aligned}
B_{i}(t) & =B_{y}(t)-\int_{0}^{t} \frac{\partial B_{y}\left(t-t^{\prime}\right)}{\partial t} \exp \left(-t^{\prime} / \tau\right) d t^{\prime} \\
& =\frac{1}{\tau} \int_{0}^{t} B_{y}\left(t-t^{\prime}\right) \exp \left(-t^{\prime} / \tau\right) d t^{\prime},
\end{aligned}
$$

where $\partial \partial t$ is replaced with $-\partial \partial t^{\prime}$ before the integration by parts. If $B_{y}\left(t-t^{\prime}\right)=0$ for $t \leq t^{\prime}$, from the convolution theorem the Laplace transform of Eq. (1) with Laplace variable $p$ is given by

$$
B_{i}(p)=B_{y}(p) \frac{1}{1+p \tau}
$$

For an inducing kicker field of a half-sine wave with a pulse width $t_{o}=\pi \omega$ and its Laplace transform,

$$
\begin{gathered}
B_{y}(t)=B_{o} \sin (\omega t), \quad 0 \leq t \leq t_{o} \\
B_{y}(p)=B_{o} \frac{\omega}{p^{2}+\omega^{2}}\left\{1+\exp \left(-p t_{o}\right)\right\}
\end{gathered}
$$

we calculate the inverse Lapace transform of Eq. (2):

$$
\begin{aligned}
B_{i}(t) & =B_{o}\left[\frac{1}{\left\{1+(\omega \tau)^{2}\right\}^{1 / 2}} \sin (\omega t-\phi)+\frac{\omega \tau}{1+(\omega \tau)^{2}} e^{-t / \tau}\right], & & \text { for } t \leq t_{o} \\
& =B_{o} \frac{\omega \tau}{1+(\omega \tau)^{2}}\left\{1+e^{t_{o} / \tau}\right\} e^{-t / \tau}, & & \text { for } t \geq t_{o}
\end{aligned}
$$

where $\phi=\tan ^{-1}(\omega \tau)$, and the time constant $\tau$ has to be calculated for the specific geometry of the circular chamber.

Table 1 lists the time constants for a few different chamber geometries. The resistances and inductances are calculated for a unit length of the chamber. The magnetic field inside the chamber is assumed to be spatially uniform under a uniform time-varying external field. For the kicker fields both perpendicular and parallel to the chamber axis, the table lists the same expression of the time constant for the circular chamber, with a vacuum permeability $\mu_{o}$ and conductivity $\sigma$, as

$$
\tau=\frac{\mu_{o} \sigma a d}{2}=\frac{\mu_{o} a}{2 R_{s}}
$$


where the surface resistivity, $R_{s}=1 / \sigma d$, assumes that the coating thickness $d$ is less than the classical skin depth $\delta$. For $\sigma=1 \times 10^{-6} \mathrm{~S} / \mathrm{m}$ and $\omega=1 \times 10^{6}$, for example, $\delta$ is approximately 0.4 mm compared to $d=2 \times 10^{-3} \mathrm{~mm}$ for $R_{s}=0.05 \Omega$ [3].

Figure 2 shows the magnetic fields inside a circular beam chamber, relative to the kicker field, calculated from Eq. (5) for the time constants of the chamber, $0.25 \mu \mathrm{s}, 0.5 \mu \mathrm{s}$, and $1 \mu \mathrm{s}$. The kicker field is a half-sine wave with a pulse-width $4 \mu \mathrm{s}\left(\omega=\pi / 4 \times 10^{6}\right)$. The peak field for $\tau$ $=1 \mu \mathrm{s}$ is approximately 0.815 of that for the applied kicker field with a phase delay of $0.8 \mu \mathrm{s}$.

\section{Circular Beam Chamber}

\section{a. Kicker Magnetic Field Perpendicular to the Chamber Axis}

For the inducing kicker magnetic field applied perpendicular to the chamber axis, as shown in Fig. 1, the eddy currents in the chamber are only in the direction of the chamber axis as assumed in the previous section. This would be the case when the chamber is relatively long compared to the diameter of the chamber, and the eddy currents near both ends of the chamber are neglected. Then, the vector potential of the field, which induces the eddy currents in the conducting wall, must be parallel to the chamber axis.

We choose a circular cylindrical coordinate system $(r, \theta, z)$ such that the $\mathrm{z}$-axis coincides with the axis of the chamber. Then, from the Maxwell's equations we have:

$$
\begin{aligned}
& \nabla^{2} A_{z}(r, \theta, t)=-\mu J_{z}(r, \theta, t), \\
& E_{z}(r, \theta, t)=-\frac{\partial A_{z}(r, \theta, t)}{\partial t},
\end{aligned}
$$

where $\mu$ is the permeability of the chamber and the displacement current is neglected for a pulse width on the order of $10^{-6} \mathrm{~s}$. Since the vector potential $A(r, \theta, t)$ has the $z$-component only and does not depend on the $\mathrm{z}$-coordinate, from now on the subscript $\mathrm{z}$ for the vector potential will be omitted for brevity.

In order to have $B_{r}=\mu_{o} H_{y}(t) \sin \theta$ and $B_{\theta}=\mu_{o} H_{y}(t) \cos \theta$ for $r \rightarrow \infty$, we may express the vector potential $A_{o}$ for the outer region of the chamber, $r \geq b$, as

$$
A_{o}=-\mu_{o}\left\{H_{y}(t) r+\frac{D}{r}\right\} \cos \theta
$$

where $\mu_{o} H_{y}(t)$ is the applied kicker field and $D$ is a constant to be determined from boundary conditions. If we assume that the field in the inner region of the chamber, $r \leq a$, is uniform for uniform surface resistivity of the chamber, the potential $A_{i}$ for $r \leq a$ may be written

$$
A_{i}=-\mu_{o} H_{i}(t) r \cos \theta
$$


Combining Eqs. (7) and (8) for the chamber region $a \leq r \leq b$ with a permeability $\mu$ and conductivity $\sigma$, the equation for the vector potential $A$ we want to solve is

$$
\frac{1}{r} \frac{\partial}{\partial r}\left(r \frac{\partial A}{\partial r}\right)+\frac{1}{r^{2}} \frac{\partial^{2} A}{\partial \theta^{2}}=\mu \sigma \frac{\partial A}{\partial t} .
$$

Writing the vector potential for the chamber region in the form of

$$
A=R(r, t) \cos (n \theta)
$$

and substituting it to Eq. (11), we have the Laplace transform of the equation for $R(r, t)$

$$
\frac{d^{2} R(r, p)}{d r^{2}}+\frac{1}{r} \frac{d R(r, p)}{d r}-\left(q^{2}+\frac{n^{2}}{r^{2}}\right) R(r, p)=0
$$

with $q=(\mu \sigma p)^{1 / 2}$. From Eqs. (9) and (10), it is obvious that we have to choose the circular harmonic number $n=1$ for the spatially uniform kicker field. Hence, the solution of Eq. (13) is given by

$$
R(r, p)=F I_{1}(q r)+G K_{1}(q r)
$$

where $I_{l}(q r)$ and $K_{l}(q r)$ are modified Bessel functions of the first and second kinds of the first order, and $F$ and $G$ are constants to be determined. From the boundary conditions of the magnetic flux densities and the magnetic intensities at $r=a$ and $b$, and using the relations

$$
\frac{1}{r} \frac{\partial A}{\partial \theta}=\mu H_{r} \quad \text { and } \quad \frac{\partial A}{\partial r}=-\mu H_{\theta}
$$

for the vector potentials, $A_{o}$, $A$, and $A_{i}$, we finally have the field inside the chamber, $H_{i}(p)$ :

with

$$
H_{i}(p)=2 \mu H_{o}(p) b\left[K_{1}(q a) I_{1}^{\prime}(q a)-I_{1}(q a) K_{1}^{\prime}(q a)\right] / \Delta(q),
$$

$$
\begin{array}{r}
\Delta(q)=\mu_{o} a b\left[I_{1}^{\prime}(q a) K_{1}^{\prime}(q b)-K_{1}^{\prime}(q a) I_{1}^{\prime}(q b)\right]+\mu a\left[I_{1}^{\prime}(q a) K_{1}(q b)-K_{1}^{\prime}(q a) I_{1}(q b)\right] \\
+\mu b\left[K_{1}(q a) I_{1}^{\prime}(q b)-I_{1}(q a) K_{1}^{\prime}(q b)\right]+\mu^{2} / \mu_{o}\left[K_{1}(q a) I_{1}(q b)-I_{1}(q a) K_{1}(q b)\right]
\end{array}
$$

where the prime denotes $K_{1}^{\prime}(q a)=d K_{1}(q r) /\left.d r\right|_{r=a}$.

For a wall thickness of chamber $d=b-a$, much smaller than the classical skin depth, Eq. (16) may be approximated, after the Taylor expansion of $I_{l}(q r)$ and $K_{l}(q r)$ near $r=a$ or $b$ to the first order in $d$. Then using recurrence and Wronskian relations for the modified Bessel functions, we have: 


$$
H_{i}(p)=\frac{2 \mu H_{o}(p)}{a d \mu_{o} q^{2}+2 \mu+\mu \frac{d}{a}\left(\mu_{r}+\frac{1}{\mu_{r}}-2\right)},
$$

where $\mu_{r}=\mu / \mu_{o}$. The inverse Laplace transform of Eq. (17) for the kicker field of a half-sine wave (Eq. (3)) and its Laplace transform (Eq. (4)) give the same form as Eq. (5) but with a different expression for the time constant $\tau$ :

$$
\tau=\frac{\mu_{o} \sigma a d}{2\left\{1+(d / a)\left(\mu_{r}-1\right)^{2} / \mu_{r}\right\}} .
$$

For $\mu_{r}=1$, Eq. (18) is reduced to Eq. (6). It should be noted that when $\mu_{r}$ is on the order of $a / d\left(10^{3}\right.$, for example), the time constant reduces to approximately one-half of Eq. (6). When the kicker field has to be expressed in terms of the $n$th circular harmonics, the solution for Eq. (13) may be given by a linear combination of order $n$ of the modified Bessel functions, and the time constant will be reduced to $\tau / n$.

\section{b. Kicker Magnetic Field Parallel to the Chamber Axis}

When the chamber axis is not perfectly aligned perpendicular to the kicker field, there will be a field component parallel to the axis, $\mu_{o} H_{z}(t)$. Let us assume that the field penetrated inside the chamber, $\mu_{o} H_{i}(t)$, is parallel to the axis. This requires that the surface resistivity of the chamber be relatively uniform so that the eddy current due to $\mu_{o} H_{z}(t)$ in the region of the chamber, $a \leq r \leq$ $b$, be in the azimuth direction. After the Laplace transform of the following Maxwell's equations in the cylindrical coordinates,

$$
\frac{1}{r} \frac{\partial}{\partial r}\left(r E_{\theta}\right)=-\mu \frac{\partial H_{z}}{\partial t} \text { and } \quad-\frac{\partial H_{z}}{\partial r}=\sigma E_{\theta}
$$

the equation to be solved for $H_{z}$ is given by

$$
\frac{\partial^{2} H_{z}(r, p)}{\partial r^{2}}+\frac{1}{r} \frac{\partial H_{z}(r, p)}{\partial r}-q^{2} H_{z}(r, p)=0,
$$

where $q$ is the same parameter defined for Eq. (13). The solution may be expressed in terms of order zero of the modified Bessel functions, $I_{0}(q r)$ and $K_{0}(q r)$ :

$$
H_{z}(r, p)=f I_{0}(q r)+g K_{0}(q r)
$$

Using Eq. (19), we have the following relations for the magnetic field, $\mu_{o} H_{i}$, for the region $r \leq a$ : 


$$
\begin{aligned}
& E_{\theta}(a)=-\mu a p H_{i}(p), \\
& \sigma E_{\vartheta}(a)=-\frac{\partial}{\partial r}\left[f I_{o}(q r)+g K_{o}(q r)\right]_{r=a} .
\end{aligned}
$$

Together with the continuity conditions for $H_{z}(r, p)$ at $r=a$ and $b$, we obtain

$$
\frac{H_{i}(p)}{H_{z}(p)}=\frac{2\left[I_{0}(q a) K_{0}^{\prime}(q a)-K_{0}(q a) I_{0}^{\prime}(q a)\right]}{a q^{2}\left[I_{0}(q a) K_{0}(q b)-K_{0}(q a) I_{0}(q b)\right]+2\left[I_{0}(q b) K_{0}^{\prime}(q a)-K_{0}(q b) I_{0}^{\prime}(q a)\right]}
$$

The approximation made for Eq. (16) can be applied to Eq. (20) to find

$$
H_{i}(p)=\frac{2 H_{z}(p)}{a d q^{2}+2}
$$

and the inverse transform of which, for a half-sine wave pulse, gives the same results as Eq. (5) with a time constant $\tau$

$$
\tau=\frac{1}{2} \mu \sigma a d
$$

For $\mu_{r}=1$, the time constants for $H_{z}(t)$ and $H_{y}(t)$ are the same. For $\mu_{r}>1$, it should be noted that the time constant for $H_{z}(t)$ increases with $\mu_{r}$, while that for $H_{y}(t)$ decreases with $1 /\left(2+\mu_{r} d / a\right)$.

\section{Elliptic Beam Chamber}

For a beam chamber of elliptic cross section as shown in Fig. 3, we use elliptic coordinates:

$$
\begin{array}{ll} 
& w=\xi+i \eta=\cosh ^{-1}(z / h), \\
\text { or } & z=x+i y=h(\cosh \xi \cos \eta+i \sinh \xi \sin \eta), \\
\text { with } & h_{\xi}=h_{\eta}=|d z / d w|=h / 2^{1 / 2}(\cosh 2 \xi-\cos 2 \eta)^{1 / 2} .
\end{array}
$$

A constant value of $\xi$ represents a confocal ellipse with major and minor axes, $2 h \cosh \xi$ and $2 h \sinh \xi$, and foci at $(x, y)=( \pm h, 0)$.

When the kicker magnetic field $\mu_{o} H_{y}(t)$ is parallel to the minor axis of the chamber, the equation to be solved for the vector potential $A$ (subscript $z$ is omitted for brevity) for the chamber region $\xi_{1} \leq \xi \leq \xi_{2}$ in Fig. 3 is given by

$$
\frac{1}{h_{\xi}^{2}} \frac{\partial^{2} A}{\partial \xi^{2}}+\frac{1}{h_{\eta}^{2}} \frac{\partial^{2} A}{\partial \eta^{2}}=\mu \sigma \frac{\partial A}{\partial t}
$$


After the Laplace transform, the desired form for the vector potential may be expressed as $A(\xi, \eta, q)=\Xi(\xi, q) \Theta(\eta, q)$. Then we have two Mathieu equations:

$$
\frac{d^{2} \Theta(\eta)}{d \eta^{2}}+[c+2 q \cos (2 \eta)] \Theta(\eta)=0
$$

and $\frac{d^{2} \Xi(\xi)}{d \xi^{2}}-[c+2 q \cosh (2 \xi)] \Xi(\xi)=0$

where $c$ is the arbitrary separation constant and $q=\left(h q_{1} / 2\right)^{2}$ is a positive parameter with $q_{1}=(\mu \sigma p)^{1 / 2}$.

Outside of the chamber region $\xi \geq \xi_{2}$, where $q=0$, we should have $c=n^{2}$ ( $n$ : odd integers) in order to have a periodicity of $2 \pi$ for the vector potential, $A(\xi, \eta)=A(\xi, \eta+2 \pi)$. Additional conditions of

$$
H_{\xi}=H_{y}(p) \sin \eta, \quad H_{\eta}=H_{y}(p) \cos \eta, \quad(\xi \rightarrow \infty)
$$

and relations $\quad \frac{1}{h_{\eta}} \frac{\partial A}{\partial \eta}=\mu H_{\xi}, \quad-\frac{1}{h_{\xi}} \frac{\partial A}{\partial \xi}=\mu H_{\eta}$,

require that $n=1$, and the vector potential $A_{o}$ for the outside may be written as

$$
A_{o}(\xi, \eta, p)=-h \mu_{o} H_{y}(p) \cosh \xi \cos \eta+\sum_{n=1,3, \cdots} a_{n}(p) e^{-n \xi} \cos (n \eta)
$$

where $a_{n}(p)$ are constants to be determined. Inside the chamber $\xi \leq \xi_{1}$, the vector potential and the magnetic field components $\mu_{o} H_{\eta}$ and $\mu_{o} H_{\xi}$, must be continuous near both sides of the interfocal line $(\xi \rightarrow 0)$ :

$$
\begin{aligned}
& \left.A_{i}(\xi, \eta)\right|_{\xi \rightarrow 0}=\left.A_{i}(\xi,-\eta)\right|_{\xi \rightarrow 0}, \\
& \left.\frac{\partial}{\partial \xi} A_{i}(\xi, \eta)\right|_{\xi \rightarrow 0}=-\left.\frac{\partial}{\partial \xi} A_{i}(\xi,-\eta)\right|_{\xi \rightarrow 0},\left.\quad \frac{\partial}{\partial \eta} A_{i}(\xi, \eta)\right|_{\xi \rightarrow 0}=-\left.\frac{\partial}{\partial \eta} A_{i}(\xi,-\eta)\right|_{\xi \rightarrow 0}
\end{aligned}
$$

Then the only permissible form of $A_{i}$, with the magnetic field at the chamber axis $\mu_{o} H_{i}(t)$ may be written as

$$
A_{i}(\xi, \eta, p)=-h \mu_{o} H_{i}(p) \sum_{n=1,3, \cdots} b_{n}(p) \cosh (n \xi) \cos (n \eta)
$$


where $n$ has to be odd integers to have the periodicity of $2 \pi$ in $\eta$, and $b_{n}$ are constants to be determined.

For the region of the chamber wall $\xi_{1} \leq \xi \leq \xi_{2}$, the solutions for Eq. (24) are Mathieu functions by writing $-q$ for $q$ or $(\pi / 2-\xi)$ for $\xi$. Here we choose only the cosine series for $\Theta(\eta, q)$ :

$$
c e_{1}(\eta,-q)=\sum_{r=0}^{\infty}(-1)^{r} B_{2 r+1}^{1} \cos (2 r+1) \eta
$$

where the series $B_{2 r+1}^{1}$ is a function of $q$. The solutions for Eq. (25), by writing $(\pi \mathrm{i} / 2+\eta)$ for $\eta$, are modified Mathieu functions of the first kind for $q$ negative. Hence, the function $\Xi(\xi)$ may be expressed in terms of two independent series of the modified Bessel functions [4]:

$$
\begin{aligned}
\Xi(\xi, q) & =f^{\prime} \operatorname{Cce}_{1}(\eta,-q)+g^{\prime} \text { Fek }_{1}(\eta,-q) \\
& =\sum_{r=0}^{\infty}(-1)^{r} B_{2 r+1}^{1}\left[f I_{2 r+1}(2 \sqrt{q} \cosh \xi)+g K_{2 r+1}(2 \sqrt{q} \cosh \xi)\right],
\end{aligned}
$$

where $f$ and $g$ are constants to be determined. For $h \rightarrow 0, \xi \rightarrow \infty$, and $h \cos h \xi \rightarrow a$, the argument $2 \sqrt{q} \cosh \xi$ approaches $q_{1} a$ in Eq. (31), and the terms for $r=0$ converge to Eq. (14) for the circular chamber.

\section{References}

[1] S. Milton and D. Warner, “The APS Ceramic Chambers," EPAC'94, p. 2494 (1994).

[2] W. R. Smythe, Static and Dynamic Electricity, McGraw-Hill, New York, 2nd Ed., p. 411 (1950).

[3] C. Doose, private communication.

[4] N. W. McLachlin, Theory and Application of Mathieu Functions, Dover Publication, Inc., New York (1964). 
Table 1. Time constants of magnetic field decays for a few different chamber geometries. The coating of the chamber wall is assumed to have a uniform surface resistivity $R_{s}=$ $1 / \sigma d$. The applied magnetic field outside the chamber is spatially uniform (see Figs. 1 and 3 ). The field direction is perpendicular to the chamber axis for all chamber geometries except Chamber Geometry $b$.

\begin{tabular}{|l|c|c|c|}
\hline \hline \multicolumn{1}{|c|}{ Chamber Geometry } & $\underline{\mathrm{R}}$ & $\underline{\mathrm{L}}$ & $\underline{\tau=\mathrm{L} / \mathrm{R}}$ \\
\hline a. Circular, radius $a$ & $\frac{2}{\pi \sigma a d}$ & $\frac{\mu_{o}}{\pi}$ & $\frac{\mu_{o} \sigma a d}{2}$ \\
\hline $\begin{array}{l}\text { b. Circular, radius } a \text {, field } \\
\text { parallel to the axis }\end{array}$ & $\frac{2 \pi a}{\sigma d}$ & $\pi \mu_{o} a^{2}$ & $\frac{\mu_{o} \sigma a d}{2}$ \\
\hline $\begin{array}{c}\text { c. Rectangular, width } w \\
\begin{array}{c}\text { d. Elliptic, major axis } 2 a, \\
\text { minor axis } 2 b\end{array}\end{array} \frac{\frac{2}{\sigma w d}}{\pi \sigma a d} \mathrm{f}_{1}$ & $\frac{4 \mu_{o}}{\pi}$ & $\frac{2 \mu_{o} \sigma w d}{\pi}$ \\
\hline$(b / a)^{2}=0.75$ & $\mathrm{f}_{1}=1.07$ & $\mathrm{f}_{2}=1.10$ & $\frac{\mu_{o} \sigma a d}{2} \mathrm{f}_{3}$ \\
\hline 0.5 & 1.15 & 1.26 & 1.03 \\
\hline 0.25 & 1.26 & 1.55 & 1.23 \\
\hline 0.1 & 1.35 & 1.97 & 1.46 \\
\hline
\end{tabular}

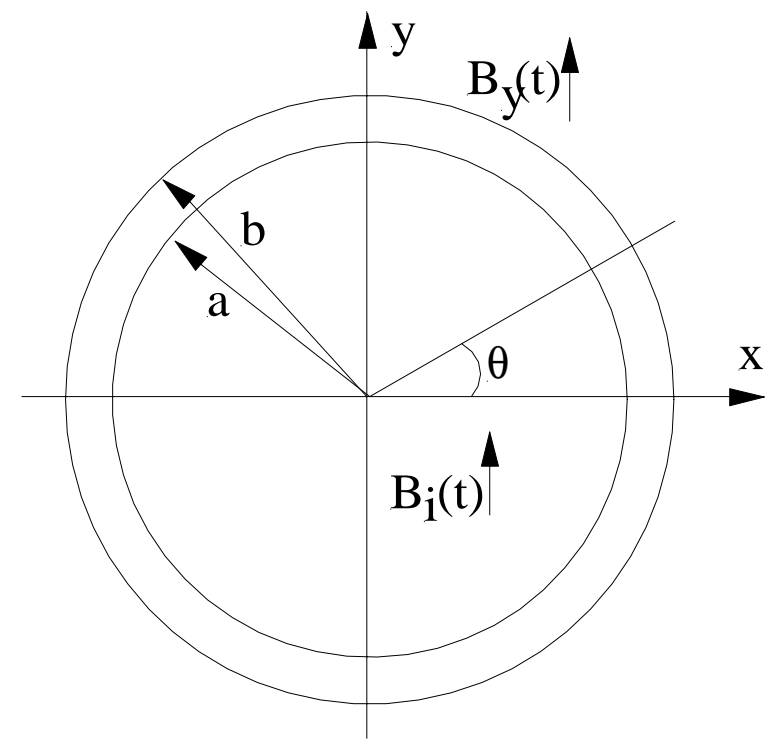

Fig. 1. Cross section of a circular chamber in a pulsed vertical kicker magnetic field $B_{y}(t)$. The chamber wall thickness $d=b-a$, which is greatly exaggerated in the figure compared to the radius, is a coating of conducting material. $B_{i}(t)$ denotes field penetrated inside the chamber. The positive $\mathrm{x}$-axis is the reference for the azimuthal angle $\theta$ of the cylindrical coordinate system $(r, \theta, z)$. 


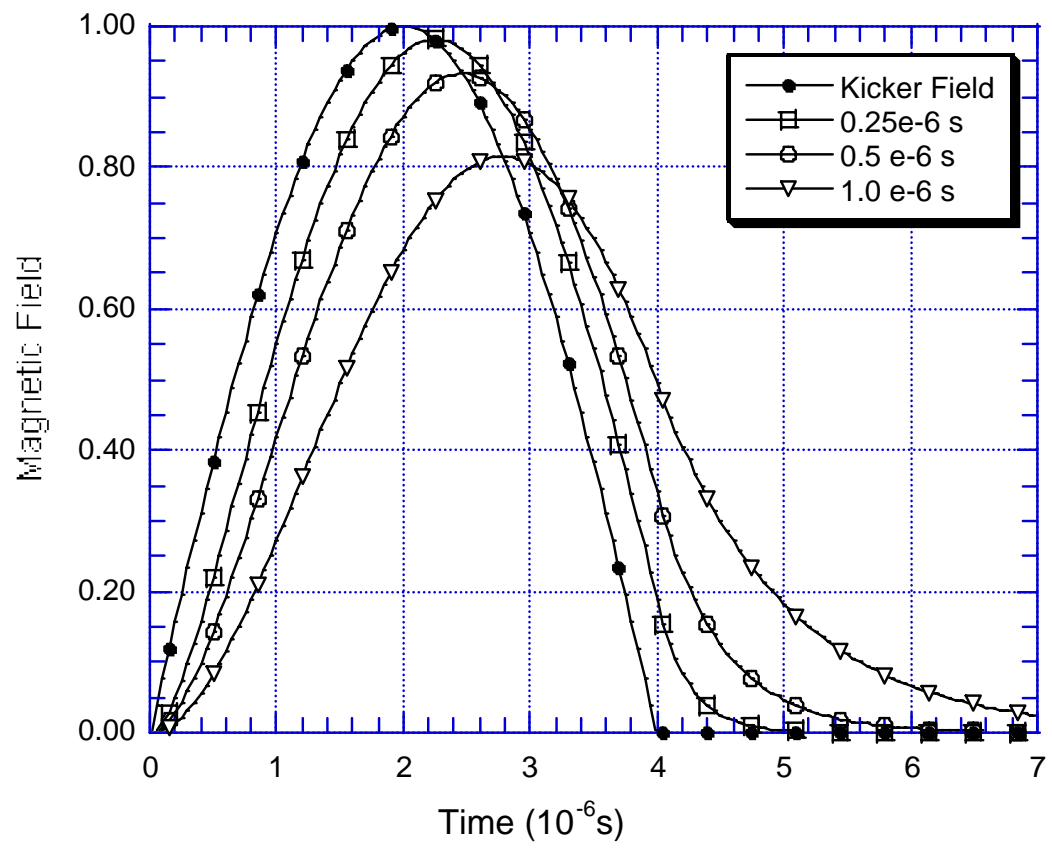

Fig. 2. Magnetic fields inside a circular chamber calculated from Eq. (5) for the time constants $0.25 \mu \mathrm{s}, 0.5 \mu \mathrm{s}$, and $1 \mu \mathrm{s}$. The magnetic fields are relative to the kicker field of a half-sine wave of a $4-\mu$ s pulse-width.

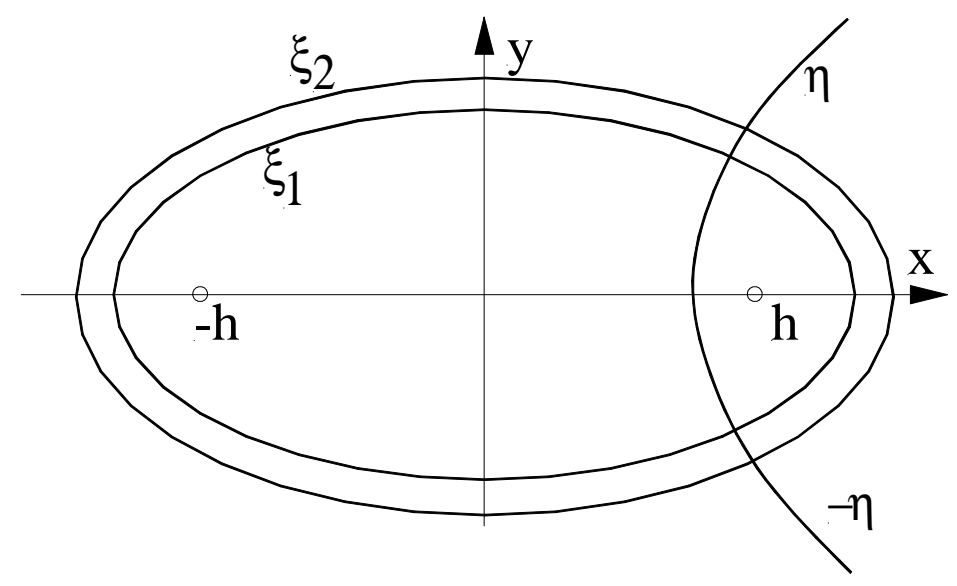

Fig. 3. Cross section of an elliptic chamber in a pulsed vertical kicker magnetic field $B_{y}(t)$. The chamber wall thickness $\xi_{2}-\xi_{1}$, which is greatly exaggerated in the figure compared to the minor or major axes, is a coating of conducting material. The foci are located at $(x, y)=( \pm h, 0)$. 